\title{
Cell Culture Pooling
}

National Cancer Institute

\section{Source}

National Cancer Institute. Cell Culture Pooling. NCI Thesaurus. Code C112952.

The expansion of a cell line in multiple vessels followed by the harvesting and pooling of the expanded culture into one vessel for holding or storage. 